\title{
ORDER-COMPATIBLE TOPOLOGIES ON A PARTIALLY ORDERED SET
}

\author{
E. S. WOLK
}

1. Introduction. Let $X$ be a partially ordered set (poset) with respect to a relation $\leqq$, and possessing least and greatest elements 0 and $I$ respectively. There are many known ways of using the order properties of $X$ to define an "intrinsic" topology on $X$. It is our purpose in this note, instead of considering certain special topologies of this type, to introduce a class of topologies on $X$ which are compatible, in a natural sense, with its order. To this end, let us call a subset $S$ of $X$ up-directed (down-directed) if and only if for all $x \in S$ and $y \in S$ there exists $z \in S$ with $z \geqq x, z \geqq y(z \leqq x, z \leqq y)$. Also, following McShane [3], we shall call a subset $K$ of $X$ Dedekind-closed if and only if whenever $S$ is an up-directed subset of $K$ and $y=1$.u.b. $(S)$, or $S$ is a down-directed subset of $K$ and $y=$ g.l.b. $(S)$, we have $y \in K$. We now introduce the following definition, which seems to be a natural requirement for a topology on $X$ to be harmoniously related to its order structure.

Definition. If $J$ is a topology defined on $X$, we shall say that $J$ is order-compatible with $X$ if and only if

(i) every set closed with respect to $J$ is Dedekind-closed, and

(ii) every set of the form $\{x \in X \mid a \leqq x \leqq b\}$ is closed with respect to 3 .

The main purpose of this note is to obtain a simple sufficient condition for a poset $X$ to possess a unique order-compatible topology. We say that two elements $x$ and $y$ in $X$ are incomparable if and only if $x \leq y$ and $x \geq y$. Let us call a subset $S$ of $X$ diverse if and only if $x \in S$, $y \in S$, and $x \neq y$ imply that $x$ and $y$ are incomparable. We define the width of $X$ to be the l.u.b. of the set $\{k \mid k$ is the cardinal number of a diverse subset of $X\}$. We shall then prove, as our main result, that a poset of finite width possesses a unique order-compatible topology, with respect to which it is a Hausdorff topological space.

2. Preliminary definitions and lemmas. The reader may verify that the class of all Dedekind-closed subsets of a poset $X$ is closed with respect to arbitrary intersections and finite unions. Hence we may define a topology $D$ on $X$ whose closed sets are precisely the Dedekindclosed subsets of $X$. We let $g$ denote the well-known interval topology on $X$, which is obtained by taking all sets of the form $[a, b]$

Received by the editors February 15, 1958. 
$=\{x \mid a \leqq x \leqq b\}$ as a sub-basis for the closed sets. If $\mathcal{S}$ and $J$ are any topologies on $X$, we define $\delta \leqq J$ to mean that every $\delta$-closed set is I-closed. It is then obvious that we have

Lemma 1. If $J$ is any order-compatible topology on $X$, then $\mathfrak{g} \leqq \mathfrak{J} \leqq \mathcal{D}$.

Lemma 2. If $X$ contains no infinite diverse set, then $X$ is a Hausdorff space in its interval topology.

Proof. Suppose $a$ and $b$ are any distinct points of $X$. Then [4] $X$ is a Hausdorff space in its interval topology if there is a covering of $X$ by means of a finite number of closed intervals such that no interval contains both $a$ and $b$. We consider the following cases, and produce such a covering in each instance.

Case (i). $a$ and $b$ are incomparable. Let $S$ be a maximal diverse subset of $X$ containing both $a$ and $b$. Consider all intervals of the form $[0, s]$ and $[s, I]$ for $s \in S$. This is a finite set of intervals satisfying the above requirements.

Case (ii). $a<b$, but $a<x<b$ for no $x \in X$. Let $S$ be a maximal diverse subset of $X$ containing $a$, and let $T$ be a maximal diverse set containing $b$. Consider the following collections of intervals:

(1) all intervals of the form $[0, s]$ for $s \in S$,

(2) all intervals of the form $[t, I]$ for $t \in T$,

(3) all intervals which may exist of the form $[s, t]$ for $s \in S$ and $t \in T$, provided that $s=a$ and $t=b$ are not both true.

The union of the above three collections of intervals satisfies our requirements.

Case (iii). $a<b$ and there exists $x_{0}$ with $a<x_{0}<b$. Let $S$ be a maximal diverse subset containing $x_{0}, T$ a maximal diverse subset containing $b$. Then the union of the following three collections of intervals satisfies our requirements:

(1) all intervals of the form $[0, s]$ for $s \in S$,

(2) all intervals of the form $[t, I]$ for $t \in T$,

(3) all intervals which may exist of the form $[s, t]$ for $s \in S, t \in T$.

Since the above three cases dispose of all possibilities, the proof is complete.

We shall find it convenient to consider nets of elements in $X$. We shall follow the terminology of Bartle [1] and Kelley [2], but give all the relevant definitions. If $f$ is a function defined on an arbitrary up-directed poset $A$ and with values lying in $X$, then we say that $f$ is a net on $A$ to $X$. We shall use the notation $(f(\alpha), \alpha \in A)$ for such a net. A net $(g(\beta), \beta \in B)$ is said to be a subnet of $(f(\alpha), \alpha \in A)$ if and only if there is a mapping $\pi: B \rightarrow A$ which satisfies 
(i) $g(\beta)=f(\pi(\beta))$ for all $\beta \in B$, and

(ii) given any $\alpha_{0} \in A$, there exists $\beta_{0} \in B$ such that if $\beta \geqq \beta_{0}$ then $\pi(\beta) \geqq \alpha_{0}$.

Let us call a subset of $A$ of the form $A_{\beta}=\{\alpha \in A \mid \alpha \geqq \beta\}$ a residual subset of $A$. A subset $C$ of $A$ will be called cofinal in $A$ if and only if $\alpha \in A$ implies there exists $\gamma \in C$ with $\gamma \geqq \alpha$. If $f$ is a net on $A$ to $X$, and $A_{\beta}$ is a residual subset of $A$, then the net $\left(f(\alpha), \alpha \in A_{\beta}\right)$ will be called a residual subnet of $f$. If $C$ is cofinal in $A$, then the net $(f(\alpha), \alpha \in C)$ will be called a cofinal subnet of $f$. If $\beta \in A$, we shall write $E_{f}(\beta)$ (or simply $E(\beta)$, if no confusion can arise) to denote the set $\{x \in X \mid x=f(\alpha)$ for some $\alpha \geqq \beta\}$. A net $f$ on $A$ to $X$ is said to be universal if and only if given any subset $S \subset X$ then either (i) there exists $\beta \in A$ such that $E(\beta) \subset S$, or (ii) there exists $\beta \in A$ such that $E(\beta) \subset S^{\prime}$, the complement of $S$ with respect to $X$. It is a well-known result $[1 ; 2]$ that every net possesses a subnet which is universal.

Now let $J$ be any topology on $X$. We say that a net $f$ on $A$ to $X$ converges to an element $y$ in $X$ if and only if for any J-open set $U$ containing $y$, there exists $\beta \in A$ such that $E(\beta) \subset U$. If $f$ converges to $y$, we write $f(\alpha) \rightarrow y$. A subset $S$ of $X$ is closed with respect to $J$ if and only if whenever $f$ is a net whose range is in $S$ and $f(\alpha) \rightarrow y$, then $y \in S[2$, p. 66].

The following notation will be useful. If $S \subset X$, we write $S^{*}=\{x \in X \mid x \geqq s$ for all $s \in S\}$, and $S^{+}=\{x \in X \mid x \leqq s$ for all $s \in S\}$. If $f$ is a net on $A$ to $X$, let $P_{f}$ be the union of all sets of the form $\{E(\beta)\}^{+}$, for some $\beta \in A$; and let $Q_{f}$ be the union of all sets of the form $\{E(\beta)\}^{*}$, for some $\beta \in A$. Then we say that an element $y$ in $X$ is medial for $f$ if and only if $y \in P_{f}^{*} \cap Q_{f}^{+}$. We shall need the following lemma, which was proved by Ward [5, Lemma 1] using the terminology of filters.

LEMMA 3 (WARD). If $f$ is a net with range in $X$, and if $f$ converges to $y$ in the interval topology on $X$, then $y$ is medial for $f$.

3. Main results. Our main theorem will follow as a consequence of three more lemmas.

Lemma 4. Let $f$ be a net on $A$ to $X$ and suppose that $f(\alpha) \rightarrow y$ in the interval topology on $X$. If $f(\alpha)$ is incomparable with $y$ for all $\alpha \in A$, then there exists an infinite diverse subset of $X$ contained in the range of $f$.

Proof. Let $(u(\alpha), \alpha \in D)$ be a universal subnet of $f$. Since every subnet of a convergent net is convergent, and to the same limit, we 
have $u(\alpha) \rightarrow y$ in the interval topology on $X$. By Lemma 3, $y$ is medial for $u$.

We shall construct inductively an infinite diverse subset of $X$. Select $\delta_{1} \in D$ arbitrarily. Since $y \in P_{u}^{*}$ and $u\left(\delta_{1}\right)$ is incomparable with $y$, we must have $u\left(\delta_{1}\right) \notin P_{u}$. Hence the set $K_{1}=\left\{x \in X \mid x \geqq u\left(\delta_{1}\right)\right\}$ contains no $E_{u}(\alpha)$ for any $\alpha \in D$. Since $u$ is a universal net, there exists some $\alpha_{1} \in D$ such that $\alpha_{1}>\delta_{1}$ and $E_{u}\left(\alpha_{1}\right) \subset K_{1}^{\prime}=\left\{x \in X \mid x \geq u\left(\delta_{1}\right)\right\}$. Also, since $y \in Q_{u}^{+}$, we have $u\left(\delta_{1}\right) \notin Q_{u}$; and hence $L_{1}=\left\{x \in X \mid x \leqq u\left(\delta_{1}\right)\right\}$ contains no $E_{u}(\alpha)$ for any $\alpha \in D$. Hence there exists some $\beta_{1} \in D$ such that $\beta_{1}>\delta_{1}$ and $E_{u}\left(\beta_{1}\right) \subset L_{1}^{\prime}=\left\{x \in X \mid x \leq u\left(\delta_{1}\right)\right\}$. Select $\gamma_{1} \in D$ such that $\gamma_{1} \geqq \alpha_{1}, \gamma_{1} \geqq \beta_{1}$. Then $E_{u}\left(\gamma_{1}\right) \subset E_{u}\left(\alpha_{1}\right) \cap E_{u}\left(\beta_{1}\right)$. It is clear from our construction that $u\left(\delta_{1}\right)$ is incomparable with each element of $E_{u}\left(\gamma_{1}\right)$. Now choose $\delta_{2} \in D$ such that $\delta_{2} \geqq \gamma_{1}$. In an analogous way we obtain $\alpha_{2}$ and $\beta_{2}$ such that $E_{u}\left(\alpha_{2}\right) \subset\left\{x \in X \mid x \geq u\left(\delta_{2}\right)\right\}, E_{u}\left(\beta_{2}\right)$ $\subset\left\{x \in X \mid x \leq u\left(\delta_{2}\right)\right\}$, and $\alpha_{2}>\delta_{2}, \beta_{2}>\delta_{2}$. Then choose $\gamma_{2} \in D$ such that $\gamma_{2} \geqq \alpha_{2}, \gamma_{2} \geqq \beta_{2}$. Then each element of $E_{u}\left(\gamma_{2}\right)$ is incomparable with both $u\left(\delta_{1}\right)$ and $u\left(\delta_{2}\right)$. Select $\delta_{3} \geqq \gamma_{2}$. Continuing in the above manner we obtain an infinite sequence of distinct elements $u\left(\delta_{1}\right), u\left(\delta_{2}\right)$, $u\left(\delta_{3}\right), \cdots$, which form a diverse subset of $X$.

Lemma 5. Let $f$ be a net on $A$ to $X$, let $S$ be the range of $f$, and suppose that $y$ is medial for $f$. If $f(\alpha)<y$ for all $\alpha \in A$, then $y=1$.u.b.(S).

Proof. Suppose that there exists $z \in S^{*}$ with $z \geq y$. Since $z \in\left\{E_{f}(\alpha)\right\}^{*}$ for all $\alpha \in A$, we have $z \in Q_{f}$. But $y \in Q_{f}^{+}$, and hence we have a contradiction.

The obvious dual formulation of the above lemma, and also that of the following one, may be left to the reader.

Lemma 6. Let $X$ be a poset of finite width, and let $f$ be a net on $A$ with range $(f)=S \subset X$. Let $y$ be an element of $X$ such that $y$ is the l.u.b. of the range of every subnet of $f$. Then there exists an up-directed set $M \subset S$ such that $y=1 . u$.b. $(M)$.

Proof. Let $k=$ width of $X$. Let us suppose that the lemma is false We shall proceed to obtain a contradiction by constructing a diverse subset of $X$ containing $k+1$ elements.

It is an easy consequence of Zorn's Lemma that every up-directed subset of a poset is contained in a maximal up-directed subset. Let $M_{1}$ be any maximal up-directed subset of $S$. By our assumption that the lemma is false, we must have $y \neq 1$.u.b. $\left(M_{1}\right)$. Hence there exists no subnet of $f$ with range contained in $M_{1}$. Therefore there exists $\alpha_{1} \in A$ such that $E\left(\alpha_{1}\right) \subset S-M_{1}$. Now let us choose a maximal up-directed 
subset $M_{2}$ of $E\left(\alpha_{1}\right)$. Since by assumption there exists no subnet of ( $\left.f(\alpha), \alpha \in A_{\alpha_{1}}\right)$ with range contained in $M_{2}$, then there is an $\alpha_{2} \in A$ with $\alpha_{2}>\alpha_{1}$ and $E\left(\alpha_{2}\right) \subset E\left(\alpha_{1}\right)-M_{2}$. Now choose $M_{3}$, a maximal updirected subset of $E\left(\alpha_{2}\right)$, and continue the above process for $k$ steps. We obtain sets $M_{1}, M_{2}, \cdots, M_{k}$; and $E\left(\alpha_{1}\right), E\left(\alpha_{2}\right), \cdots, E\left(\alpha_{k}\right)$, such that (with the agreement that $\left.E\left(\alpha_{0}\right)=S\right) M_{i}$ is a maximal up-directed subset of $E\left(\alpha_{i-1}\right)$ and $E\left(\alpha_{i}\right) \subset E\left(\alpha_{i-1}\right)-M_{i}$, for $i=1,2, \cdots, k$.

Next let us note that, for each $i=1,2, \cdots, k, x \in E\left(\alpha_{i-1}\right)-M_{i}$ implies (i) $x \in M_{i}^{*}$, and (ii) $x \leq m$ for any $m \in M_{i}$. For if either (i) or (ii) failed to hold, then the set $M_{i} \cup\{x\}$ would be an up-directed subset of $E\left(\alpha_{i-1}\right)$, thus contradicting the maximality of $M_{i}$. Thus for each $x \in E\left(\alpha_{i-1}\right)-M_{i}$ there exists $x_{i} \in M_{i}$ such that $x$ and $x_{i}$ are incomparable.

Now choose an arbitrary element, which we denote by $x_{k+1}$, of $E\left(\alpha_{k-1}\right)-M_{k}$. By the above paragraph, there exists $x_{k} \in M_{k}$ such that $x_{k+1}$ and $x_{k}$ are incomparable. Also, since $x_{k} \in E\left(\alpha_{k-2}\right)-M_{k-1}$, there exist $a_{1} \in M_{k-1}$ and $a_{2} \in M_{k-1}$ such that $a_{1}$ and $x_{k}$ are incomparable, $a_{2}$ and $x_{k+1}$ are incomparable. Let $x_{k-1}$ be an element of $M_{k-1}$ with $x_{k-1} \geqq a_{1}, x_{k-1} \geqq a_{2}$. Then $x_{k-1}$ is incomparable with both $x_{k}$ and $x_{k+1}$, so that the set $\left\{x_{k+1}, x_{k}, x_{k-1}\right\}$ is diverse. Continuing in this way, we select elements $b_{1}, b_{2}, b_{3}$ in $M_{k-2}$ such that $b_{1}$ and $x_{k-1}, b_{2}$ and $x_{k}, b_{3}$ and $x_{k+1}$ form incomparable pairs. Let $x_{k-2}$ be an element of $M_{k-2}$ with $x_{k-2} \geqq b_{i}(i=1,2,3)$. Then $\left\{x_{k+1}, x_{k}, x_{k-1}, x_{k-2}\right\}$ is a diverse set. It is clear that continuing the above construction leads to a diverse set $\left\{x_{k+1}, x_{k}, \cdots, x_{1}\right\}$ of $k+1$ distinct elements, contained in range $(f)$.

We now have the following theorem.

Theorem. If $X$ is a poset of finite width, then $X$ possesses a unique order-compatible topology. Furthermore, with respect to this topology, $X$ is a Hausdorff space.

Proof. In view of Lemmas 1 and 2, we need only to prove that the topologies $\mathscr{g}$ and $D$ are equivalent on $X$. Let $K$ be any Dedekindclosed subset of $X$; we shall show that $K$ is $g$-closed. Let $f$ be a net in $K$ with $f(\alpha) \rightarrow y$ in the interval topology. We may assume that $f(\alpha) \neq y$ for all $\alpha$. We shall prove that $y \in K$. By Lemma 4 , there exists no subnet $g$ of $f$ such that each element of range $(g)$ is incomparable with $y$. Hence there exists a residual subnet of $f$, which we take to be $f$ itself, whose range consists of elements all of which are comparable with $y$. Then there exists (i) a cofinal subnet $u$ of $f$ such that $y$ is an upper bound of range $(u)$, or (ii) a cofinal subnet $v$ of $f$ such that $y$ is a lower bound of range (v). Suppose that (i) holds 
(the other case is handled in the obvious dual manner). Since $u$ converges to $y$ in the interval topology, $y$ is medial for $u$ (Lemma 3). Let $S=$ range $(u)$. By Lemma $5, y=1$.u.b. $(S)$. Since every subnet of $u$ converges to $y$ in the interval topology, Lemma 6 now applies; and we conclude that there exists an up-directed set $M \subset S \subset K$ such that $y=$ l.u.b. $(M)$. Since $K$ was assumed to be Dedekind-closed, we have $y \in K$, completing the proof.

It is natural to ask whether, in the above theorem, the hypothesis that $X$ is of finite width can be replaced by the weaker condition that $X$ contains no infinite diverse subset. However, we have not been able to settle this question (not even in the special case when $X$ is assumed to be a lattice).

\section{REFERENCES}

1. R. G. Bartle, Nets and filters in topology, Amer. Math. Monthly vol. 62 (1955) pp. 551-557.

2. J. L. Kelley, General topology, Van Nostrand, 1955.

3. E. J. McShane, Order-preserving maps and integration processes, Annals of Mathematics Studies vol. 31, Princeton, 1953.

4. E. S. Northam, The interval topology of a lattice, Proc. Amer. Math. Soc. vol. 4 (1953) pp. 825-827.

5. A. J. Ward, On relations between certain intrinsic topologies in partially ordered sets, Proc. Cambridge Philos. Soc. vol. 51 (1955) pp. 254-261.

University of ConNecticut 